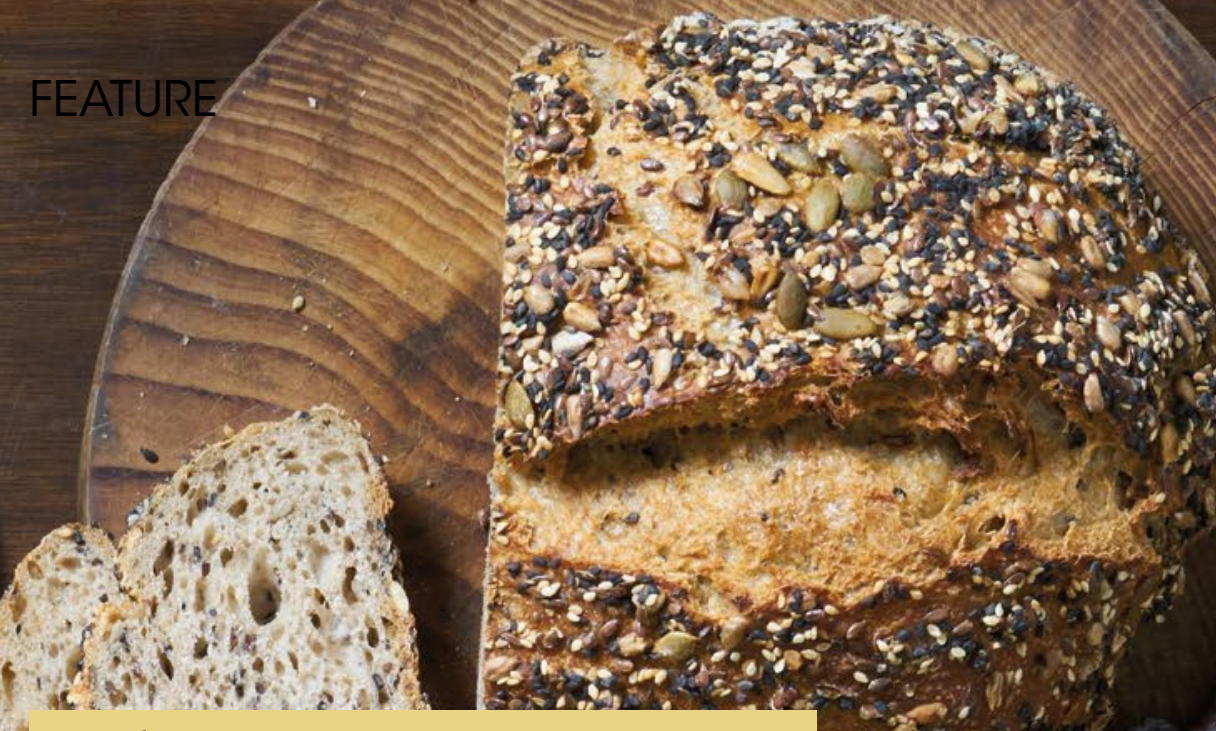

(T)

\section{The impact of}

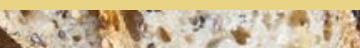

\section{a vegan diet on}

\section{oral health}

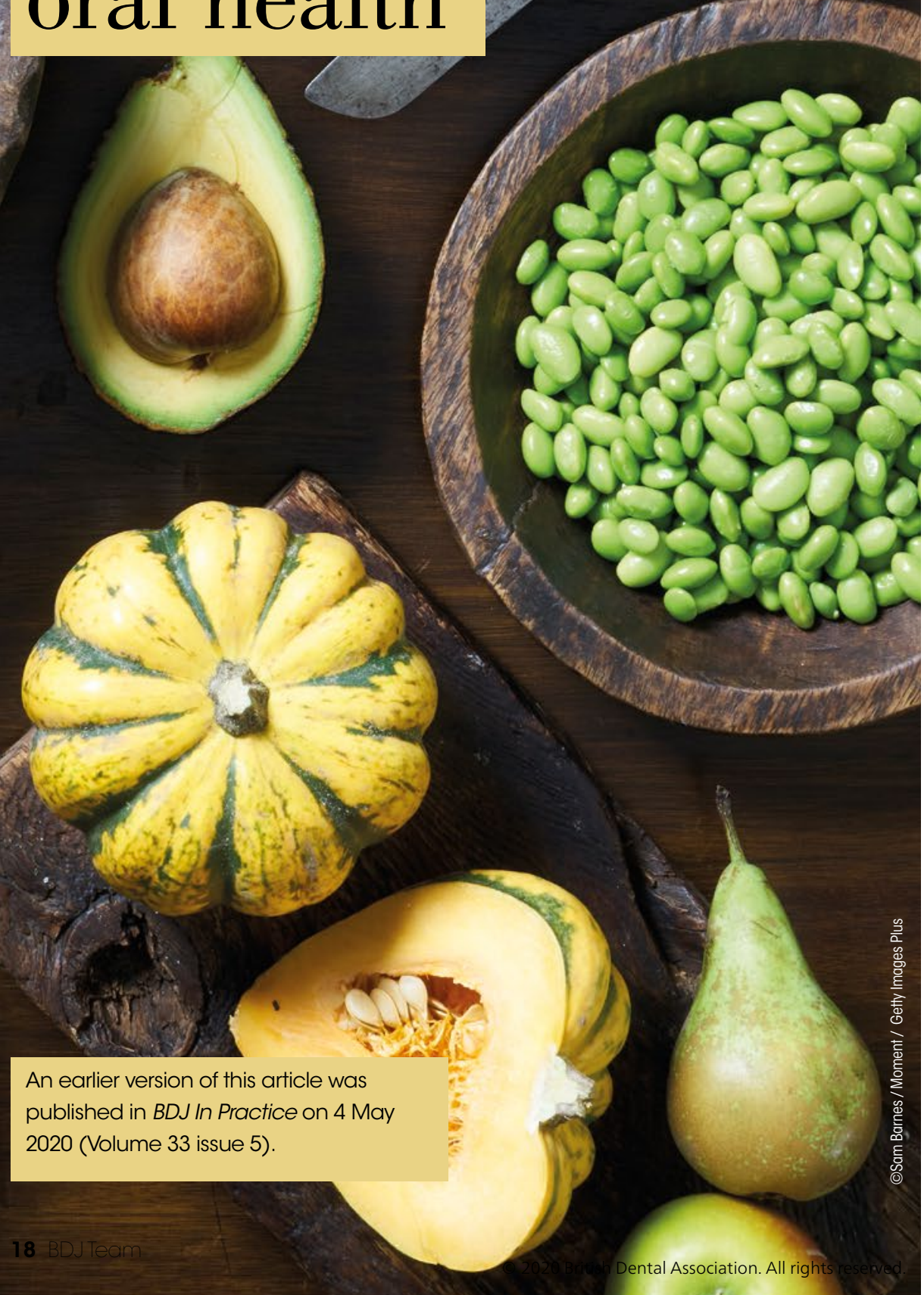

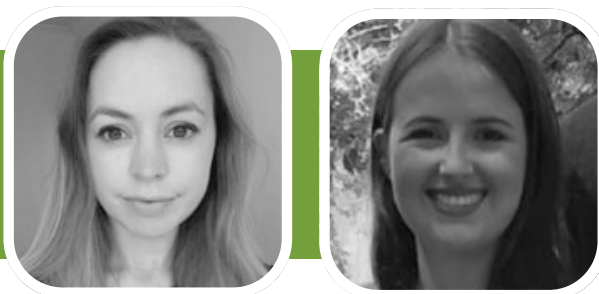

Dental foundation trainees Joelle Booth and Julia Hurry explore how a vegan diet affects oral health and the role of the dental professional.

V eganism is the practice of refraining from the use of animal products, which has a huge impact on diet as meat and dairy items are no longer consumed. With 600,000 being the estimated number of vegans in the UK in 2019 and this number quadrupling since 2014, it is more likely than ever that our patients will be consuming a plant based diet. ${ }^{1}$

Additionally, although not following a strict vegan diet, many individuals are taking the decision to reduce their consumption of meat and dairy products due to ethical, environmental and health reasons.

The aim of this article is to highlight the role of the dental professional with regards to identifying those who follow a vegan diet, discussing its impact on oral health as well as providing more tailored advice for prevention. This trend appears to be on the rise which emphasises the importance of understanding the impacts that veganism has on oral health and the delivery of dental care by dental professionals.

\section{Oral health}

Whilst research into the effect of a vegetarian diet on oral health is fairly well established, the implications of veganism on oral health is limited. Early research with small sample sizes has suggested that a vegan diet may predispose patients to demineralisation and white spot lesions. ${ }^{2}$

There are several reasons as to why this may be the case: vegans may substitute animal derived products with starchy, sugar-based carbohydrates that contribute to demineralisation, for example. Furthermore, 
to meet the recommended daily calorie consumption, those adopting a vegan diet may need to eat more frequently throughout the day, increasing the number of acid attacks within the day in comparison to their meat eating counterparts.

Studies conducted assessing those consuming a vegetarian diet have indicated that these patients experience higher levels of acid erosion than omnivores. ${ }^{3}$

It is likely that this trend could be seen in a vegan population too due to greater intake of fruit and vegetables, which in turn reduces the salivary $\mathrm{pH}$ and leads to erosive tooth surface loss. It is possible that there may also be associated dietary practices such as drinking hot water and lemon or the consumption of smoothies and juices that could accelerate tooth surface loss. This highlights the importance of monitoring tooth surface loss at regular intervals through the Basic Erosive Wear Examination (BEWE), study models/ intraoral scans and clinical photography.

It is important to gain an appreciation as to the numerous confounding factors that could be at play between a vegan diet and oral health. Those choosing to follow a vegan diet for health reasons may be by virtue more motivated, health-conscious individuals and follow health promoting practices. ${ }^{4}$ For example, they may be more likely to adhere to recommended oral hygiene advice, be nonsmokers, avoid alcohol, and be conscious of high sugar intake.

\section{Oral hygiene advice}

Understanding your patients' motivations and ethical viewpoints can assist in providing tailored and attainable oral hygiene advice.

Many large pharmaceutical companies claim to be cruelty free but do not specify whether this means their products are vegan. Glycerine, which prevents toothpaste from drying out, can be derived from either vegetable or animal sources. As one of the largest toothpaste manufacturers is set to launch their first vegan toothpaste, including vegetable based glycerine, this highlights the high demand for plant based alternatives in the market. Furthermore, companies are aiming for their products to be more sustainable and recyclable, as more consumers are driving towards ethical purchasing.

It is of concern that many vegan toothpastes currently on the market are branded as fluoride free. Following the $\mathrm{DBOH}$ guidance, ${ }^{5}$ fluoride containing toothpaste should be recommended by practitioners to all patients to reduce their risk of carious lesions. Whilst giving oral hygiene advice, emphasis should be given that fluoride itself

'We can play a key role in the holistic care of our patients and signpost them to their medical practitioner if we believe there could be a systemic link to their oral symptoms.'

is vegan friendly and vegan toothpastes containing fluoride are available.

Although a widely recommended preventative measure, many fluoride varnish products are surprisingly not vegan as they contain shellac, which is secreted from female lac bugs. Practitioners should be aware of this and ensure vegan patients are informed prior to application to obtain valid informed consent.

\section{Diet advice}

As vegan patients are at higher risk of demineralisation and acid erosion, dietary advice is key to good long term oral health.

Patients should be made aware of the hidden sugars - especially in products marketed as natural snacks - in their diet through the use of a diet diary and tailored education. Furthermore, detailed discussion should be had with vegan patients to monitor their acid intake, following advice to limit their quantity and frequency.

\section{Systemic health}

As health practitioners, it is important to discuss with our patients the impact of a vegan diet on overall health, whilst studies indicate that it can lower risks of cancer, obesity and heart disease. ${ }^{6,7}$

The NHS recommends ensuring dietary needs of iron, vitamin B12 and calcium are met through a varied diet or supplementation. Deficiencies in these nutrients could lead to oral medicine conditions such as exacerbation of recurrent aphthous ulcers and the development of glossodynia. ${ }^{8}$

Although no apparent research has been conducted into the effects of a vegan diet on periodontal health, previous studies have indicated an association between low vitamin B12 levels and increased attachment loss in patients. ${ }^{9}$ As dental professionals, we can play a key role in the holistic care of our patients and signpost them to their medical practitioner if we believe there could be a systemic link to their oral symptoms.

\section{Conclusion}

This brief overview about the impact of veganism on dentistry, particularly oral health, oral hygiene and diet advice, and general health, highlights the need for further research in this area. With veganism becoming an increasingly popular lifestyle choice, dental professionals need further guidance to better manage the needs of this patient base.

\section{References}

1. TNS-BMRB, Food Standards Agency. Food and You Survey, 2014. [data collection]. UK Data Service. Available at: http://doi.org/10.5255/UKDASN-7576-1 (accessed April 2020).

2. Laffranchi L, Zotti F, Bonetti S, Dalessandri D, Fontana P. Oral implications of the vegan diet: observational study. Minerva Stomatologica 2010; 59: 583-591.

3. Smits K P J, Listl S, Jevdjevic M. Vegetarian diet and its possible infuence on dental health: a systematic literature review. Comm Dent Oral Epidemiol 2020; 48: 7-13.

4. Mazur M, Bietolini S, Bellardini D et al. Oral health in a cohort of individuals on a plant-based diet: a pilot study. Clin Ter 2020; 171: e142-148.

5. Public Health England. Delivering better oral health: an evidence-based toolkit for prevention. 2014. Available at: https://assets. publishing.service.gov.uk/government/ uploads/system/uploads/attachment_data/ fle/605266/Delivering_better_oral_health. pdf (accessed April 2020).

6. Dinu M, Abbate R, Gensini G F, Casini A, Sof F. Vegetarian, vegan diets and multiple health outcomes: a systematic review with meta-analysis of observational studies. Crit Rev Food Sci Nutr 2017; 57: 3640-3649.

7. Craig W J. Health effects of vegan diets. Am J Clin Nutr 2009; 89: 1627S-1633S.

8. Kim J, Kim M J, Kho H S. Oral manifestations in vitamin B12 defciency patients with or without history of gastrectomy. BMC Oral Health 2016; 16: 60.

9. Zong G, Holtfreter B, Scott A E et al. Serum vitamin B12 is inversely associated with periodontal progression and risk of tooth loss: a prospective cohort study. $J$ Clin Periodontol 2016; 43: 2-9.

https://doi.org/10.1038/s41407-020-0372-3: 